\title{
GENERAL POSITION PROPERTIES SATISFIED BY FINITE PRODUCTS OF DENDRITES
}

\author{
BY
}

\author{
PHILIP L. BOWERS ${ }^{1}$
}

\begin{abstract}
Let $\bar{A}$ be a dendrite whose endpoints are dense and let $A$ be the complement in $\bar{A}$ of a dense $\sigma$-compact collection of endpoints of $\bar{A}$. This paper investigates various general position properties that finite products of $\bar{A}$ and $A$ possess. In particular, it is shown that (i) if $X$ is an $L C^{n}$-space that satisfies the disjoint $n$-cells property, then $X \times \bar{A}$ satisfies the disjoint $(n+1)$-cells property, (ii) $\bar{A}^{n} \times[-1,1]$ is a compact $(n+1)$-dimensional AR that satisfies the disjoint $n$-cells property, (iii) $\bar{A}^{n+1}$ is a compact $(n+1)$-dimensional AR that satisfies the stronger general position property that maps of $n$-dimensional compacta into $\bar{A}^{n+1}$ are approximable by both $Z$-maps and $Z_{n}$-embeddings, and (iv) $A^{n+1}$ is a topologically complete $(n+1)$-dimensional AR that satisfies the discrete $n$-cells property and as such, maps from topologically complete separable $n$-dimensional spaces into $A^{n+1}$ are strongly approximable by closed $Z_{n}$-embeddings.
\end{abstract}

1. Introduction. It is well known that the standard $(2 n+1)$-cell $I^{2 n+1}$ is a compact absolute retract that satisfies the disjoint $n$-cells property and, as such, admits an embedding of every compact $n$-dimensional metric space. Moreover, the collection of embeddings of an $n$-dimensional compactum $X$ into $I^{2 n+1}$ forms a dense $G_{\delta}$ in the function space $C\left(X, I^{2 n+1}\right)[\mathbf{H W}]$. Though there are compact $n$-dimensional spaces that satisfy the disjoint $n$-cells property and admit embeddings of every $n$-dimensional compactum, there are no topologically complete $n$ dimensional absolute neighborhood retracts that satisfy this property, for any such space would contain uncountably many pairwise disjoint embedded $n$-cells and thereby violate a result of Sieklucki [Si]. In this paper, we produce examples of compact $(n+1)$-dimensional absolute retracts that satisfy the disjoint $n$-cells property, and the collection of embeddings of an $n$-dimensional compactum $X$ into any of these examples forms a dense $G_{\delta}$ in the corresponding function space. The examples are particularly nice in that they arise as product spaces whose factors are compact 1-dimensional absolute retracts, equivalently, dendrites.

The disjoint $n$-cells property is but one example of a variety of general position properties that have played an interesting and increasingly important role in Geometric Topology during the past fifteen years, particularly in the area of characterizations of manifolds modeled on various spaces. The disjoint disks property of

Received by the editors February 6, 1984 and, in revised form, June 8, 1984.

1980 Mathematics Subject Classification. Primary 54F50; Secondary 54F35, 54C25. in $X$.

Key words and phrases. Disjoint $n$-cells property, discrete $n$-cells property, locally $n$-connected

${ }^{1}$ The results of this paper appear as part of the author's doctoral dissertation written under the direction of J. J. Walsh at the University of Tennessee [Bo1].

(C) 1985 American Mathematical Society $0002-9947 / 85 \$ 1.00+\$ .25$ per page 
J. W. Cannon is crucial in the current characterizations of finite dimensional manifolds $[\mathbf{C a}, \mathbf{E d}, \mathbf{Q u}]$ as are the disjoint and discrete cells properties of $\mathrm{H}$. Torunczyk in the current characterizations of infinite dimensional manifolds modeled on the Hilbert cube and Hilbert space $\left[\mathbf{T o}_{\mathbf{1}}, \mathbf{T o}_{\mathbf{2}}\right]$. It was during an investigation of factors of Hilbert space manifolds that the author was led to examine the various general position properties that the finite products of dendrites and special subsets of dendrites possess. The results subsequently obtained are of interest in their own right, without regard to manifold theory. The author has learned that $\mathrm{H}$. Torunczyk independently has obtained many of the results herein and in particular, the examples alluded to in the first paragraph.

A dendrite is a nondegenerate uniquely arcwise connected Peano space, equivalently, a compact 1-dimensional absolute retract. Throughout the paper, $\bar{A}$ denotes the class of dendrites whose endpoints are dense and $\bar{A}$ denotes a typical element of $\bar{A}$. In $\S 2$, we prove the following theorem.

THEOREM 2.1. If for some $n \in \mathbf{N} \cup\{0\}, X$ is an $L C^{n}$ metric space that satisfies the disjoint $n$-cells property and $\bar{A} \in \bar{A}$, then $X \times \bar{A}$ satisfies the disjoint $(n+1)$-cells property.

This theorem allows us to produce the examples alluded to in the first paragraph of this introduction.

EXAMPLE. If $n \in \mathbf{N}$ and $\bar{A} \in \bar{A}$, then $I \times \bar{A}^{n}$ satisfies the disjoint $n$-cells property. Moreover, every map of an n-dimensional compactum into $I \times \bar{A}^{n}$ can be arbitrarily closely approximated by embeddings.

If we replace the interval $I$ by $\bar{A}$ in the previous example, we obtain examples of compact $(n+1)$-dimensional absolute retracts that satisfy a stronger general position property.

COROllary 2.8. If $n \in \mathbf{N} \cup\{0\}$ and $\bar{A} \in \bar{A}$, then every map of an $n$ dimensional compactum into $\bar{A}^{n+1}$ can be arbitrarily closely approximated by both $Z$-maps and $Z_{n}$-embeddings.

For those readers who are familiar with current results in Hilbert space manifold theory, the definitions of the discrete cells properties that appear in $\S 3$ are natural extensions to the finite dimensional world of H. Torunczyk's strong discrete approximation property that appears in his characterization of Hilbert space manifolds $\left[\mathbf{T o}_{2}\right]$. As with the disjoint cells properties, the discrete cells properties have important consequences in embedding theory. The proof of the following theorem appears implicitly in $\left[\mathbf{T o}_{\mathbf{2}}\right]$.

THEOREM (TORUNCZYK). A topologically complete separable ANR X satisfies the discrete $n$-cells property if and only if every map from any topologically complete separable $n$-dimensional space into $X$ is strongly approximable by closed embeddings.

Let $A$ denote the following class of topologically complete separable 1-dimensional absolute retracts: $A$ is an element of $A$ provided $A$ is the complement in some $\bar{A}$ from $\bar{A}$ of a dense $\sigma$-compact collection of endpoints of $\bar{A}$. J. J. Walsh, the author's thesis advisor, suggested to the author that the elements of $A$ might play a role in Hilbert space manifold theory in many ways analogous to the role played by the interval $I$ in Hilbert cube manifold theory (see $\left[\mathbf{B o}_{1}\right]$ ). This led to an investigation of the general position properties satisfied by finite products 
whose factors are in $\mathcal{A}$, and the results of this investigation appear in $\S \S 3$ and 4 . In particular, in $\S 4$ we prove

COROLlary 4.3. If $n \in \mathbf{N} \cup\{0\} \cup\{\infty\}$ and $A \in A$, then $A^{n+1}$ is a topologically complete separable $A R$ that satisfies the discrete $n$-cells property.

The proof of Corollary 4.3 involves rather technical results that supply characterizations of those $\sigma$ - $Z$-sets in locally compact separable metric spaces whose complements satisfy one of the discrete cells properties. These technical results appear in $\S 3$ and are influenced by the work of D. W. Curtis that appears in $\left[\mathbf{C u}_{\mathbf{1}}\right.$, ACM, vM].

Terminology and notation. By $I$ we denote the interval $[-1,1]$, by $\mathbf{N}$ the collection of positive integers; continuous functions are called "maps." All spaces are assumed to be separable and metrizable, and if $X$ is a space, then $\rho$ usually denotes any metric on $X$ compatible with the topology on $X$. Sometimes, for emphasis, we write $(X, \rho)$ for the space $X$ with metric $\rho$. A compact space is called a compactum. Absolute neighborhood retracts, abbreviated ANR, and absolute retracts, abbreviated AR, are understood to be absolute neighborhood retracts and absolute retracts, respectively, for the class of metrizable spaces. If $K$ is an abstract simplicial complex, we use standard abuse of notation and also write $K$ for the standard geometric realization of $K$ and $K^{(n)}$ for both the abstract $n$-skeleton of $K$ and its standard geometric realization. If $f$ and $g$ are maps of a space $X$ into a space $Y$ and $U$ is an open cover of $Y$ and $\varepsilon: Y \rightarrow(0, \infty)$ is a map, then $f$ is said to be $\mathcal{U}$-close (respectively, $\varepsilon$-close) to $g$ provided for every $x \in X,\{f(x), g(x)\} \subset U$ for some $U$ in $\mathcal{U}$ (respectively, for every $x \in X, \rho(f(x), g(x))<\varepsilon(f(x))) . X \approx Y$ means $X$ is homeomorphic to $Y$. By $i, k, n$ we denote elements of $\mathbf{N} \cup\{0\} \cup\{\infty\}$ and $i<n$ means " $i \leq n-1$ if $n \neq \infty$ and $i \neq \infty$ if $n=\infty$ ", and $\infty+1$ and $\infty-1$ mean $\infty$.

2. Disjoint cells properties and products of dendrites. Recall that $\bar{A}$ denotes the class of dendrites whose endpoints are dense and $A$ denotes the class of spaces that arise as complements of dense $\sigma$-compact collections of endpoints in spaces from $\bar{A}$. If $X$ is an element of either $\bar{A}$ or $A$, then $\mathcal{E}(X)$ denotes the collection of endpoints of $X$.

A metric space $X$ is said to satisfy the disjoint $n$-cells property provided any two maps of the $n$-cell $I^{n}$ into $X$ can be arbitrarily closely approximated by maps whose images are disjoint. In [Da], R. J. Daverman proves that if an $L C^{1}$ space $X$ satisfies the disjoint 1-cells property, then $X \times I^{2}$ satisfies the disjoint 2-cells property. Theorem 2.1, stated in the introduction and proved below, illustrates the usefulness of replacing the interval $I$ by a dendrite $\bar{A}$ whose endpoints are dense.

ProOF OF THEOREM 2.1. Let $\varepsilon>0$ and let $f$ and $g$ be maps of $I^{n+1}$ into $X \times \bar{A}$. Since $X$ is $L C^{n}$ and $\bar{A}$ contains a dense set of endpoints, we can choose a finite triangulation $T$ of $I^{n+1}$ and for each $(n+1)$-simplex $\sigma$ in $T$, open sets $U_{\sigma}$ and $V_{\sigma}$ in $X$ of diameter less than $\varepsilon / 2$ and $B_{\sigma}$ and $C_{\sigma}$ in $\bar{A}$ such that $f(\sigma) \subset U_{\sigma} \times B_{\sigma}, g(\sigma) \subset V_{\sigma} \times C_{\sigma}$, maps of the $n$-sphere $S^{n}$ into $U_{\sigma}$ and $V_{\sigma}$ are $\varepsilon / 2$-homotopic to constant maps, $B_{\sigma}$ (respectively, $C_{\sigma}$ ) is $\varepsilon / 2$-contractible to an endpoint $b(\sigma)$ (respectively, $c(\sigma)$ ) in $\bar{A}$, and $\left\{b(\sigma) \mid \sigma \in T^{(n+1)}\right\} \cap\{c(\sigma) \mid \sigma \in$ $\left.T^{(n+1)}\right\}=\emptyset$. Let $f_{1}=p_{X} \circ f \mid P$ and $f_{2}=p_{\bar{A}} \circ f \mid P$ where $P$ denotes the $n$-skeleton of $T$ and $p_{X}: X \times \bar{A} \rightarrow X$ and $p_{\bar{A}}: X \times \bar{A} \rightarrow \bar{A}$ are the projection mappings, and define $g_{1}$ and $g_{2}$ similarly by replacing $f$ by $g$. Choose $\varepsilon / 2$-approximations 
$\mu$ and $\nu$ to $f_{1}$ and $g_{1}$, respectively, such that $\mu(P) \cap \nu(P)=\emptyset$ and assume that $\mu$ is so close to $f_{1}$ that $\mu(\partial \sigma) \subset U_{\sigma}$ for each $\sigma \in T^{(n+1)}$ and similarly for $\nu$. It is easy to construct a retraction $h$ of $\bar{A}$ onto a subset of $\bar{A}$ so that $h$ is $\varepsilon / 2$-close to id $\bar{A}, h\left(B_{\sigma}\right) \subset B_{\sigma}$ and $h\left(C_{\sigma}\right) \subset C_{\sigma}$ for each $\sigma \in T^{(n+1)}$, and $b(\sigma) \notin h(\bar{A})$ and $c(\sigma) \notin h(\bar{A})$ for each $\sigma \in T^{(n+1)}$. Let $f^{\prime}=\left(\mu, h \circ f_{2}\right)$ and $g^{\prime}=\left(\nu, h \circ g_{2}\right)$ and observe that $f^{\prime}, g^{\prime}: P \rightarrow X \times \bar{A}$ are maps which satisfy $f^{\prime}(P) \cap g^{\prime}(P)=\emptyset$, and $f^{\prime}(\partial \sigma) \subset U_{\sigma} \times B_{\sigma}$ and $g^{\prime}(\partial \sigma) \subset V_{\sigma} \times C_{\sigma}$ for each $\sigma \in T^{(n+1)}$.

We now define $f^{\prime}$ and $g^{\prime}$ over the $(n+1)$-skeleton of $T$. Let $\sigma$ be an $(n+1)$-simplex and write $\sigma$ as

$$
\sigma=\frac{\partial \sigma \times[0,2]}{\partial \sigma \times\{2\}}
$$

Since $\mu(\partial \sigma) \subset U_{\sigma}$, there is an $\varepsilon / 2$-homotopy $\psi: \partial \sigma \times[1,2] \rightarrow X$ of $\mu \mid \partial \sigma$ to a point in $X$. Since $h \circ f_{2}(\partial \sigma) \subset B_{\sigma}$, there is an $\varepsilon / 2$-homotopy $\theta: \partial \sigma \times[0,1] \rightarrow \bar{A}$ of $h \circ f_{2} \mid \partial \sigma$ to the endpoint $b(\sigma)$ of $\bar{A}$. Since for each $\tau \in T^{(n+1)}, c(\tau) \notin h \circ f_{2}(\partial \sigma)$, it can be arranged that $c(\tau) \notin \theta(\partial \sigma \times[0,1])$. Use $[s, t]$ for $(s, t) \in \partial \sigma \times[0,2]$ to denote a point of $\sigma$ and define $f^{\prime}$ on $\sigma$ by

$$
f^{\prime}([s, t])= \begin{cases}(\mu(s), \theta(s, t)), & 0 \leq t \leq 1, \\ (\psi(s, t), b(\sigma)), & 1 \leq t \leq 2 .\end{cases}
$$

$f^{\prime}$ is well defined and continuous on $\sigma$ and extends $f^{\prime} \mid \partial \sigma$. Similarly, with $\nu, V_{\sigma}, h \circ$ $g_{2}, C_{\sigma}$, and $c(\sigma)$ respectively in place of $\mu, U_{\sigma}, h \circ f_{2}, B_{\sigma}$, and $b(\sigma)$, define $g^{\prime}$ on $\sigma$. In this way we obtain maps $f^{\prime}, g^{\prime}: I^{n+1} \rightarrow X \times \bar{A}$ and it is easy to see that $p_{X} \circ f^{\prime}$ is $\varepsilon$-close to $p_{X} \circ f$ and $p_{\bar{A}} \circ f^{\prime}$ is $\varepsilon$-close to $p_{\bar{A}} \circ f$ and similarly for $g^{\prime}$. Also, it is straightforward to verify that $f^{\prime}\left(I^{n+1}\right) \cap g^{\prime}\left(I^{n+1}\right)=\emptyset$ and thus $X \times \bar{A}$ satisfies the disjoint $(n+1)$-cells property.

The main fact that allows us to prove Theorem 2.1 is that $\bar{A}$ contains a dense collection of endpoints. An easy Baire category argument shows that every element of $A$ also contains a dense collection of endpoints (specifically, if $F$ is a $\sigma$-compact subset of $\mathcal{E}(\bar{A})$, then $\mathcal{E}(\bar{A})-F$ is dense in $\bar{A}$ ) and this allows us to prove the following corollary using exactly the same proof as above.

Corollary 2.2. The previous theorem is true with $A \in A$ in place of $\bar{A} \in \bar{A}$.

According to [To $\mathbf{T o}_{\mathbf{1}}$, if $X$ is a topologically complete ANR that satisfies the disjoint $n$-cells property, then every map of a compact $n$-dimensional metric space into $X$ can be arbitrarily closely approximated by embeddings. In fact, the collection of embeddings of any $n$-dimensional compactum into $X$ forms a dense $G_{\delta}$-subset of the corresponding function space. The following corollary provides examples of $(n+1)$-dimensional AR's that are universal for $n$-dimensional compacta in the sense of this paragraph.

Corollary 2.3. If $\bar{A} \in \bar{A}, A \in A$, and $n \in \mathbf{N}$, then $I \times \bar{A}^{n}, I \times A^{n}, \bar{A}^{n+1}$, and $A^{n+1}$ satisfy the disjoint $n$-cells property.

Proof. $I, \bar{A}$, and $A$ satisfy the disjoint 0-cells property. Apply Theorem $2.1 n$ times.

COROLlary 2.4. Let $X$ denote either $I \times \bar{A}^{n}, I \times A^{n}, \bar{A}^{n+1}$, or $A^{n+1}$ where $\bar{A} \in \bar{A}, A \in A$, and $n \in \mathbf{N}$. Then the collection of embeddings of an $n$-dimensional compactum $Y$ into $X$ forms a dense $G_{\delta}$-subset of $C(Y, X)$. 
In [DW], R. J. Daverman and J. J. Walsh prove that if $X \times Y$ is a manifold modeled on the Hilbert cube, abbreviated $Q$-manifold, for a locally compact space $X$ and a finite dimensional space $Y$, then $X \times I^{2}$ is a $Q$-manifold. Theorem 2.1 provides an improvement of this result by replacing the 2-dimensional AR $I^{2}$ by a 1-dimensional AR $\bar{A}$.

COROLlARY 2.5. If $X \times Y$ is a $Q$-manifold for a locally compact $A N R X$ and a finite dimensional space $Y$ and $\bar{A} \in \bar{A}$, then $X \times \bar{A}$ is a $Q$-manifold.

ProOF. Apply Theorem 2.1 of this paper along with Corollaries 6.3 and 6.4 of $[\mathbf{D W}]$.

The $(n+1)$-dimensional AR's $\bar{A}^{n+1}$ and $A^{n+1}$ satisfy stronger general position properties than the disjoint $n$-cells property, namely, maps of $I^{n}$ into $\bar{A}^{n+1}$ and $A^{n+1}$ can be arbitrarily closely approximated by both $Z$-maps and $Z_{n}$-embeddings. This is proved by showing that both $\bar{A}^{n+1}$ and $A^{n+1}$ contain $\sigma-Z$-sets that absorb $n$-cells. The remainder of this section outlines a proof and several corollaries of this result.

A closed subset $F$ of a space $X$ is a $Z_{n}$-set in $X$ for some $n \in \mathbf{N} \cup\{0, \infty\}$ if each map $f: I^{n} \rightarrow X$ can be arbitrarily closely approximated by maps into $X-F$. It is easy to see that $F$ is a $Z_{\infty}$-set in $X$ if and only if $X$ is a $Z_{n}$-set for all $n \in \mathbf{N}$. We use the more common term $Z$-set for $Z_{\infty}$-set. A $\sigma$ - $Z$-set is a countable union of $Z$ sets and a $Z$-map (respectively, $Z_{n}$-embedding) is a map (respectively, embedding) $f: Y \rightarrow X$ such that $f(Y)$ is a $Z$-set (respectively, $Z_{n}$-set) in $X$.

A subset $F$ of a space $X$ is said to absorb $n$-complexes for some nonnegative integer $n$ if each map $f: K \rightarrow X$ of a compact $n$-complex $K$ into $X$ can be arbitrarily closely approximated by maps whose images lie in $F$. If $F$ is a $\sigma$ - $Z$-set in $X$ that absorbs $n$-complexes, then every map of a compact $n$-complex into $X$ can be arbitrarily closely approximated by $Z$-maps.

Given $\bar{A} \in \bar{A}$ and a dense $\sigma$-compact subset $F_{0}$ of $\mathcal{E}(\bar{A})$, let $F_{1}=\left(F_{0} \times \bar{A}\right) \cup(\bar{A} \times$ $\left.F_{0}\right) \subset \bar{A}^{2}$ and, inductively, let $F_{n}=\left(F_{n-1} \times \bar{A}\right) \cup\left(\bar{A}^{n} \times F_{0}\right) \subset \bar{A}^{n+1}$. Note that $\bar{A}-F_{0}=A$ is an element of $A$ and $F_{n}=\bar{A}^{n+1}-A^{n+1}$.

LEMMA 2.6. If for some $n \in \mathbf{N} \cup\{0\}, F$ is a subset of an $L C^{n}$ metric space $X$ that absorbs $n$-complexes, then $(F \times \bar{A}) \cup\left(X \times F_{0}\right) \subset X \times \bar{A}$ absorbs $(n+1)$-complexes.

Proof. Let $f: K \rightarrow X \times \bar{A}$ be a map of a compact $(n+1)$-complex $K$ into $X \times \bar{A}$ and let $f_{1}$ and $f_{2}$ be $f$ followed by projection to $X$ and $\bar{A}$, respectively. Name a triangulation $T$ of $K$ having small mesh and let $P$ denote its $n$-skeleton. If the mesh of $T$ is small enough, we can approximate $f_{1} \mid P$ by a map $\mu: P \rightarrow F$ so that for each $\sigma \in T^{(n+1)}, \mu \mid \partial \sigma$ is homotopic via a small homotopy to a constant map and $f_{2} \mid \partial \sigma$ is homotopic via a small homotopy to an endpoint $b(\sigma)$ in $F_{0}$ (for details, see the proof of Theorem 2.1). Now extend $\left(\mu, f_{2} \mid P\right)$ to each $(n+1)$-simplex $\sigma$ in $T$ exactly as in the proof of Theorem 2.1 and thereby obtain a map $f^{\prime}$ that approximates $f$. If $z \in \sigma \in T^{(n+1)}$, then either $p_{X} \circ f^{\prime}(z)=\mu(z) \in F$ or $p_{\bar{A}} \circ f^{\prime}(z)=b(\sigma) \in F_{0}$ where $p_{X}$ and $p_{\bar{A}}$ are the obvious projections. Thus $f^{\prime}(K) \subset(F \times \bar{A}) \cup\left(X \times F_{0}\right)$ and $(F \times \bar{A}) \cup\left(X \times F_{0}\right)$ absorbs $(n+1)$-complexes.

Proposition 2.7. For $n \in \mathbf{N} \cup\{0\}, F_{n}$ is a $\sigma$-compact $\sigma$-Z-set in $\bar{A}^{n+1}$ that absorbs $n$-complexes. 
PROOF. It is an easy matter to verify that a compact subset of $\mathcal{E}(\bar{A})$ is a $Z$-set in $\bar{A}$. Thus $F_{0}$ is a $\sigma$ - $Z$-set in $\bar{A}$ and since $F_{0}$ is dense in $\bar{A}, F_{0}$ absorbs 0 -complexes. Assume that $F_{n-1}$ satisfies the conclusion of the proposition. Then easily $F_{n}=\left(F_{n-1} \times \bar{A}\right) \cup\left(\bar{A}^{n} \times F_{0}\right)$ is a $\sigma$-Z $Z$-set in $\bar{A}^{n+1}$ and an application of Lemma 2.6 with $X=\bar{A}^{n}$ and $F=F_{n-1}$ implies that $F_{n}$ absorbs $n$-complexes.

Almost immediately, we have the proof of Corollary 2.8 that is stated in the introduction.

ProOF OF COROLlaRY 2.8. Since $\bar{A}^{n+1}$ is an ANR, every map of an $n$ dimensional compactum into $\bar{A}^{n+1}$ can be arbitrarily closely factored through a compact $n$-complex. Proposition 2.7 then implies that any such map can be arbitrarily closely approximated by a $Z$-map. To obtain an approximation by a $Z_{n}$-embedding, first move the given map off of the $\sigma$ - $Z$-set $F_{n}$ by a small move, then use Corollary 2.4 to approximate this map by an embedding whose image lies in $A^{n+1}=\bar{A}^{n+1}-F_{n}$. By Proposition 2.7 (or Corollary 2.10), this image is a $Z_{n}$-set in $\bar{A}^{n+1}$.

COROllary 2.9. The previous corollary is true with $A \in A$ in place of $\bar{A} \in \bar{A}$.

Proof. Let $F_{0}^{\prime} \subset \mathcal{E}(A)$ be a dense $\sigma$-compact subset of $A$ and define $F_{n}^{\prime}$ analogously to $F_{n}$. The proofs of Lemma 2.6 and Proposition 2.7 apply to show that $F_{n}^{\prime}$ is a $\sigma$ - $Z$-set in $A^{n+1}$ that absorbs $n$-complexes.

Compact subsets of the pseudo-interior $s$ of the Hilbert cube $I^{\infty}$ are $Z$-sets in both $s$ and $I^{\infty}$. The following corollary points out that the pair $\left(\bar{A}^{n+1}, A^{n+1}\right)$ satisfies the analogous finite dimensional property.

Corollary 2.10. Let $A \in \mathcal{A}$ and let $\bar{A}$ be the corresponding element of $\bar{A}$. If $n \in \mathbf{N} \cup\{0\}$, then compact subsets of $A^{n+1}$ are $Z_{n}$-sets in both $A^{n+1}$ and $\bar{A}^{n+1}$.

The proof is immediate from Proposition 2.7.

3. Discrete cells properties. The strong discrete approximation sroperty was introduced by $\mathrm{H}$. Torunczyk in his study of Hilbert space manifolds [ $\left.\mathbf{T o}_{\mathbf{2}}\right]$ and this extremely useful approximation property subsequently has been studied by $\mathrm{R}$. D. Anderson, D. W. Curtis, and J. van Mill in $[\mathbf{A C M}]$, D. W. Curtis in $\left[\mathbf{C u}_{\mathbf{1}}\right]$, and T. Dobrowolski and H. Torunczyk in [DT]. The purpose of this section is to define finite dimensional versions of the strong discrete approximation property and to supply sufficient conditions on a dense $\sigma$-compact subset $F$ of a locally compact ANR $X$ to ensure that $X-F$ satisfies various ones of these finite dimensional versions. In the next section, we use the results of this section to investigate the various discrete cells properties satisfied by finite products of elements of $A$. Throughout this section we fix an element $n$ of $\mathbf{N} \cup\{0\} \cup\{\infty\}$.

H. Torunczyk has obtained the following topological characterization of Hilbert space manifolds $\left[\mathbf{T o}_{\mathbf{2}}\right]$.

THEOREM (TORUNCZYK). A topologically complete separable ANR $X$ is a Hilbert space manifold if and only if every map $f: \bigoplus_{i \in \mathbf{N}} I_{i}^{\infty} \rightarrow X$ of the countable free union of Hilbert cubes into $X$ is strongly approximable by maps $g: \bigoplus_{i \in \mathbf{N}} I_{i}^{\infty} \rightarrow$ $X$ for which the collection $\left\{g\left(I_{i}^{\infty}\right)\right\}_{i \in \mathbf{N}}$ is discrete.

The above approximation property is referred to as the strong discrete approximation theory in $[\mathbf{A C M}]$; however, we shall delete the adjective strong and refer 
to this as the discrete approximation property. Recall that a collection $D$ of subsets of $X$ is discrete provided every point in $X$ has a neighborhood that meets at most one element of $D$. If we replace the countable free union of Hilbert cubes by the countable free union of $n$-cells, we obtain a hierarchy of discrete cells properties:

DEFINITION 3.1. A metric space $X$ satisfies the discrete $n$-cells property (discrete $\infty$-cells property $=$ discrete approximation property) provided either of the following equivalent conditions is satisfied:

3.1.1. For each map $f: \bigoplus_{i \in \mathbf{N}} I_{i}^{n} \rightarrow X$ and each open cover $\mathcal{U}$ of $X$, there exists a map $g: \bigoplus_{i \in \mathbf{N}} I_{i}^{n} \rightarrow X$ such that $f$ and $g$ are $\mathcal{U}$-close and $\left\{g\left(I_{i}^{n}\right)\right\}_{i \in \mathbf{N}}$ is discrete.

3.1.2. For each map $f: \bigoplus_{i \in \mathbb{N}} I_{i}^{n} \rightarrow X$ and each map $\varepsilon: X \rightarrow(0, \infty)$, there exists a map $g: \bigoplus_{i \in \mathbf{N}} I_{i}^{n} \rightarrow X$ such that $f$ and $g$ are $\varepsilon$-close and $\left\{g\left(I_{i}^{n}\right)\right\}_{i \in \mathbf{N}}$ is discrete.

The following theorem is due to $\mathrm{H}$. Torunczyk and establishes the relationship between discrete cells properties and embedding theory. All of the ingredients for its proof appear in $\left[\mathbf{T o}_{\mathbf{2}}\right]$.

THEOREM (TORUNCZYK). A topologically complete separable ANR X satisfies the discrete $n$-cells property if and only if every map from any topologically complete separable $n$-dimensional space into $X$ is strongly approximable by closed embeddings.

The following theorem provides a characterization of those $\sigma$ - $Z$-sets in a locally compact separable metric space whose complements satisfy one of the discrete cells properties. The reader should note that if $X-F$ satisfies either of the equivalent properties in the theorem, then necessarily $F$ is dense in $X$.

THEOREM 3.2. Let $F$ be a $\sigma$-Z-set in a locally compact separable metric space $(X, \rho)$ and let $n \in \mathbf{N} \cup\{0\} \cup\{\infty\}$. The following statements are equivalent:

(1.n) $X-F$ satisfies the discrete $n$-cells property (discrete $\infty$-cells property $=$ discrete approximation property).

(2.n) For each map $\varepsilon: X-F \rightarrow(0, \infty)$, there exists a closed in $X$ subset $J \subset F$ such that for every map $f: I^{n} \rightarrow X-F$ and every neighborhood $N(J)$ of $J$ in $X$, there exists a map $f^{\prime}: I^{n} \rightarrow N(J)$ such that $\rho\left(f, f^{\prime}\right)<\varepsilon \circ f$.

Proof. (2.n) implies (1.n): Let $\varepsilon: X-F \rightarrow(0, \infty)$ and $f: \bigoplus_{i} D_{i} \rightarrow X-F$ be maps where for each $i \in \mathrm{N}, D_{i} \approx I^{n}$, and set $f_{i}=f \mid D_{i}$. Let $J$ be as hypothesized in (2.n) for the map $\frac{1}{2} \varepsilon$ and let $\beta(1)=\frac{1}{4} \rho\left(g_{1}\left(D_{1}\right), J\right)$ where $g_{1}=f_{1}$. According to $(2 . n)$, we can choose a map $f_{2}^{\prime}: D_{2} \rightarrow N_{\beta(1)}(J)$ (for a positive number $\beta, N_{\beta}(J)$ denotes the $\beta$-neighborhood of $J$ in $X)$ such that $\rho\left(f_{2}, f_{2}^{\prime}\right)<\frac{1}{2} \varepsilon \circ f_{2}$. Since $F$ is a $\sigma$ $Z$-set in $X$, and therefore a $\sigma-Z_{n}$-set in the topologically complete space $X$, there is a map $g_{2}: D_{2} \rightarrow X-F$ which is $\gamma_{2}$-close to $f_{2}^{\prime}$ where $\gamma_{2}=\frac{1}{2} \min \left[\{\beta(1)\} \cup \varepsilon\left(f_{2}\left(D_{2}\right)\right)\right]$. Note that $g_{2}\left(D_{2}\right) \subset N_{2 \beta(1)}(J)$ and for each $x \in D_{2}, \rho\left(f_{2}(x), g_{2}(x)\right)<\varepsilon\left(f_{2}(x)\right)$. Inductively we let $\beta(i)=\frac{1}{4} \rho\left(g_{i}\left(D_{i}\right), J\right)$ and

$$
\gamma_{i+1}=\frac{1}{2} \min \left[\{\beta(i)\} \cup \varepsilon\left(f_{i+1}\left(D_{i+1}\right)\right)\right]
$$

and obtain a map $g_{i+1}: D_{i+1} \rightarrow X-F$ which satisfies $g_{i+1}\left(D_{i+1}\right) \subset N_{2 \beta(i)}(J)$ and for each $x \in D_{i+1}, \rho\left(f_{i+1}(x), g_{i+1}(x)\right)<\varepsilon\left(f_{i+1}(x)\right)$. Let $g=\bigoplus_{i} g_{i}: \bigoplus_{i} D_{i} \rightarrow$ $X-F$ and observe that $\rho(f, g)<\varepsilon \circ f$. Since $\beta(i+1)<\frac{1}{2} \beta(i), \beta(i) \rightarrow 0$ as $i \rightarrow \infty$ 
and, since $g\left(D_{i+1}\right) \subset N_{2 \beta(i)}(J)$ and $J$ is closed in $X$, it follows easily from the definition of $\beta(i)$ that $\left\{g\left(D_{i}\right)\right\}_{i \in \mathrm{N}}$ is discrete in $X-F$.

(1.n) implies (2.n): Let $\varepsilon: X-F \rightarrow(0, \infty)$ be a map. Choose a cover of $X-F$ by relatively compact sets open in $X$ and let $Y$ denote the union of this cover. Let $W$ be a locally finite refinement of a double star refinement of this cover of $Y$ and for each $x \in Y$, let $g(x)=\rho(x, Y-\operatorname{st}(x, \mathcal{W}))$ where $\rho(x, \phi)$ is defined to be $\infty$. Thus $N_{g(x)}(x)$ is the largest ball about $x$ contained in $\operatorname{st}(x, \mathcal{W})$. Clearly $0<g(x)$ for each $x \in Y$ and we show that $g$ is a lower semicontinuous function of $Y$ into the extended reals. Let $x \in Y$ so that $g(x)>\alpha>0$ and let

$$
\beta \pm=\frac{g(x) \pm \alpha}{2} \quad(\beta \pm \text { may be } \infty) .
$$

$V=\bigcap\{W \in \mathcal{W} \mid x \in W\}$ is open in $Y$ since $\mathcal{W}$ is locally finite. Check that for each $y \in V \cap N_{\beta-}(x), N_{\beta+}(y) \subset N_{g(x)}(x) \subset \operatorname{st}(x, W) \subset \operatorname{st}(y, W)$ so that $g(y) \geq$ $\beta+>\alpha$ and thus $g$ is lower semicontinuous. Since $0<g$, Theorem $4.3[\mathrm{Du}, \mathrm{p}$. 171] applies to give a continuous function $\bar{\varepsilon}: Y \rightarrow(0, \infty)$ such that $0<\bar{\varepsilon}<g$. Let $\delta: X-F \rightarrow(0, \infty)$ be the map given by $\delta(x)=\min \{\varepsilon(x), \bar{\varepsilon}(x)\}$ and observe that $N_{\delta(x)}(x) \subset \operatorname{st}(x, W)$ for each $x \in X-F$. We leave it to the reader to show that $N_{\delta}(C)=\bigcup\left\{N_{\delta(c)}(c) \mid c \in C\right\}$ is a relatively compact subset of $Y$ for each compact $C \subset X-F$.

Since the function space $C\left(I^{n}, X-F\right)$ with metric topology is separable [Du, Theorem 5.2, p. 265 and Theorem 8.2(3), p. 270], we can choose a countable dense collection $\left\{f_{i}\right\}_{i \in \mathbf{N}}$ of maps of $I^{n}$ into $X-F$. For each $i, j \in \mathbf{N}$, define $f_{i, j}=f_{i}$ and use the fact that $X-F$ satisfies the discrete $n$-cells property to obtain maps $g_{i, j}: I^{n} \rightarrow X-F$ such that $\rho\left(f_{i}, g_{i, j}\right)<\frac{1}{2} \delta \circ f_{i}$ and $\left\{g_{i, j}\left(I^{n}\right) \mid i, j \in \mathbf{N}\right\}$ is discrete in $X-F$. Let $J$ be the "limit points" of the maps $g_{i, j}$ in $X$, that is, $x \in X$ is in $J$ if and only if there are points $x(i, j) \in g_{i, j}\left(I^{n}\right)$ such that every neighborhood of $x$ in $X$ contains infinitely many of the $x(i, j)$ 's. Since $\left\{g_{i, j}\left(I^{n}\right)\right\}$ is discrete in $X-F, J \subset F$ and it is easy to see that $J$ is closed in $X$.

Let $f: I^{n} \rightarrow X-F$ be a map and let $N(J)$ be a neighborhood of $J$ in $X$. Since $\left\{f_{i}\right\}_{i \in \mathbf{N}}$ is dense in $C\left(I^{n}, X-F\right)$ and $I^{n}$ is compact, there exists an $i \in \mathbf{N}$ such that $\left|\delta \circ f-\delta \circ f_{i}\right|<\frac{1}{2} \delta_{f}\left[\mathbf{D u}\right.$, Theorem 2.1(2), p. 259] and $\rho\left(f, f_{i}\right)<\frac{1}{4} \delta_{f}$ where $\delta_{f}=\min \left[\delta\left(f\left(I^{n}\right)\right)\right]$. Observe that for each $j \in \mathbf{N}$ and $x \in I^{n}$,

$$
\begin{aligned}
\rho\left(f(x), g_{i, j}(x)\right) & \leq \rho\left(f(x), f_{i}(x)\right)+\rho\left(f_{i}(x), g_{i, j}(x)\right) \\
& \leq \frac{1}{4} \delta_{f}+\frac{1}{2} \delta\left(f_{i}(x)\right) \leq \frac{1}{4} \delta_{f}+\frac{1}{2}\left(\frac{1}{2} \delta_{f}+\delta(f(x))\right) \leq \delta(f(x)) .
\end{aligned}
$$

Thus all we need do is to show that there exists a $j \in \mathbf{N}$ such that $g_{i, j}\left(I^{n}\right)\llcorner N(J)$. But this follows easily from the fact that $N_{\delta}\left(f_{i}\left(I^{n}\right)\right)$ is relatively compact in $X$ and contains $g_{i, j}\left(I^{n}\right)$ for each $j \in \mathbf{N}$, for otherwise there are "limit points" of $\left\{g_{i, j}\right\}_{j \in \mathbf{N}}$ which are not in $N(J)$.

For a space $X$ local compactness in Theorem 3.2 is used only for the implication (1.n) implies (2.n). The other implication is true in the setting of topologically complete separable spaces and thus provides a method for detecting those $\sigma$ - $Z$-sets in this setting whose complements satisfy a discrete cells property.

COROLlaRY 3.3. (2.n) implies (1.n) in Theorem 3.2 provided $X$ is topologically complete. 
ProOF. The proof is exactly the proof that (2.n) implies (1.n) in Theorem 3.2 except that here we hypothesize that $X$ is topologically complete whereas this is a consequence of local compactness in Theorem 3.2.

We now define two versions of what it means for a subset of a space to be locally $n$-connected in that space. We then state the precise relationship between the discrete cells properties and these two versions of local connectivity and this provides us with the tools that we use in $\S 4$ in our examination of the general position properties that products of elements from $A$ possess.

DEFINITION 3.4. For $n<\infty$, a subset $F$ of a space $X$ is locally $n$-connected in $X$ provided for every $x \in X$ and neighborhood $U$ of $x$ in $X$, there exists a neighborhood $V$ of $x$ in $X$ such that every map $f: S^{n} \rightarrow V \cap F$ is null-homotopic in $U \cap F$. We say that $F$ is $L C^{n}$ in $X$ if it is locally $i$-connected in $X$ for $0 \leq i \leq n$, and $F$ is $L C^{\infty}$ in $X$ if it is locally $i$-connected in $X$ for all $i \geq 0 . F$ is said to be $L C^{-1}$ in $X$ provided $F$ is dense in $X$.

The property of being $L C^{n}$ in $X$ is not an intrinsic property of the subset $F$ of $X$. Rather, this property combines an intrinsic property of $F$ with a property of the particular embedding of $F$ into $X$. Indeed, $F$ is $L C^{n}$ in $X$ if and only if $F$ is an $L C^{n}$ space and $X-F$ is an $L C C^{n}$ subset of $X$, the former property being an intrinsic property of $F$ and the latter a property of the embedding of $F$ into $X$. For example, $(0,1) \approx\left\{e^{i t} \in S^{1} \mid 0<t<1\right\}$ is $L C^{0}$ in $S^{1}$ while $(0,1) \approx\left\{e^{2 \pi i t} \in S^{1} \mid 0<t<1\right\}$ is not $L C^{0}$ in $S^{1}$.

We now define a continuum version of local connectivity in a space. This definition reflects the fact that a subset $F$ of a space $X$ may fail to have nice local connectivity properties, yet it may be embedded in the overlying space $X$ in such a way that nice local connectivity properties abound for open neighborhoods of $F$. This definition is inspired by Curtis $\left[\mathbf{C u}_{\mathbf{1}}\right]$ and arises naturally in the study of discrete cells properties (see $\left[\mathbf{B o}_{\mathbf{1}}\right.$ and $\left.\mathbf{C u}_{\mathbf{1}}\right]$ ).

DEFINITION 3.5. For $n<\infty$, a subset $F$ of a space $X$ is proximately locally $n$-connected in $X$ provided for every $x \in X$ and neighborhood $U$ of $x$ in $X$, there exists a neighborhood $V$ of $x$ in $X$ such that, for every compactum $S \subset V \cap F$, there exists a compactum $K \subset U \cap F$ such that, for every neighborhood $N(K)$ of $K$ in $X$, there exists a neighborhood $N(S)$ of $S$ in $X$ such that every map $f: S^{n} \rightarrow N(S)$ is null-homotopic in $N(K)$. We say that $F$ is proximately $L C^{n}$ in $X$ if it is proximately locally $i$-connected in $X$ for $0 \leq i \leq n$, and $F$ is proximately $L C^{\infty}$ in $X$ if it is proximately locally $i$-connected in $X$ for all $i \geq 0$.

If we require $X$ to be an ANR and replace "for every $x \in X$ " by "for every $x \in F$ " in Definition 3.5, we arrive at Curtis' definitions of proximately locally n-connected, proximately $L C^{n}$, and proximately $L C^{\infty}$. These properties are intrinsic properties of $F$, independent of the particular embedding of $F$ into any ANR. The property of being proximately $L C^{n}$ in $X$ holds the same relationship to being proximately $L C^{n}$ as the property of being $L C^{n}$ in $X$ holds to being $L C^{n}$. Indeed, $F$ is proximately $L C^{n}$ in $X$ if and only if $F$ is a proximately $L C^{n}$, an intrinsic property of $F$, and $F$ is $(n+1)$-target dense embedded in $X$ (maps of $(n+1)$-cells into $X$ can be pushed close to $F$ by small moves). See Curtis $\left[\mathbf{C u}_{\mathbf{1}}\right]$.

In investigating the general position properties that products of elements of $A$ possess (§4), only (i) and (iii) of Theorem 3.6 below are used. (ii) of Theorem 3.6 is included for completeness, since it provides a characterization in terms of local 
connectivity of those dense $\sigma$ - $Z$-sets in locally compact metric spaces whose complements satisfy the discrete $n$-cells property while (iii) provides merely a sufficient condition on such $\sigma$ - $Z$-sets. Recall conditions (1.n) and (2.n) from Theorem 3.2.

THEOREM 3.6. Let $F$ be a dense $\sigma$-Z-set in a locally compact separable metric space $(X, \rho)$. If $n \in \mathbf{N} \cup\{0\}$, then the following statements hold:

(i) Condition (1.n) is equivalent to condition (2.n).

(ii) If $X$ is $L C^{n-1}$, then condition (2.n) holds if and only if $F$ is proximately $L C^{n-1}$ in $X(n \neq 0)$. false.

(iii) If $F$ is $L C^{n-1}$ in $X$, then condition (2.n) holds. The reverse implication is

ProOF. (i) This is Theorem 3.2.

(ii) (2.n) implies $F$ is proximately $L C^{n-1}$ in $X$. Let $x \in X$ and let $U$ be a neighborhood of $x$ in $X$. Choose a $\delta$-ball $N_{\delta}$ about $x$ such that $\mathrm{Cl}_{X}\left(N_{3 \delta}\right)$ is compact and contained in $U$ where $N_{3 \delta}$ is the $3 \delta$-neighborhood of $x$, and choose a neighborhood $V$ of $x$ in $X$ such that maps of $S^{i}$ for $0 \leq i<n$ into $V$ are nullhomotopic in $N_{\delta}$. Let $S \subset V \cap F$ be compact and let $\varepsilon: X \rightarrow\left[0, \frac{1}{2} \delta\right]$ be a map such that $\varepsilon^{-1}(0)=S$. Let $J$ be as promised in (2.n) for the map $\varepsilon \mid X-F$ and assume that $S \subset J$. Define $K=J \cap \mathrm{Cl}_{X}\left(N_{3 \delta}\right)$, a compact subset of $U \cap F$, and let $N(K)$ be a neighborhood of $K$ in $X$.

Since $X$ is $L C^{n-1}$ and locally compact and $\varepsilon^{-1}(0)=S$, we can choose $\gamma$ with $\delta>\gamma>0$, a neighborhood $N(S)$ of $S$ in $X$, and a relatively compact open set $W$ in $X$ such that $N(S) \subset W \subset \mathrm{Cl}_{X} W \subset N(K) \cap V, \varepsilon(y)<\gamma$ for each $y \in N(S)$, and the $\gamma$-neighborhood of $N(S)$ is contained in $W$, and $\gamma$-close maps into $W$ of spheres of dimension less than $n$ are homotopic in $N(K)$.

Let $0 \leq i<n$ and let $f: S^{i} \rightarrow N(S)$ be a map. Since $F$ is a $\sigma$ - $Z$-set in $X$ and $X$ is $L C^{n-1}, f$ is homotopic in $N(K)$ to a map $g: S^{i} \rightarrow N(S)-F$ and since $N(S) \subset V$, there exists an extension $G: I^{i+1} \rightarrow N_{\delta}$ of $g$. Again since $F$ is a $\sigma$ $Z$-set and since $g\left(S^{i}\right) \cap F=\emptyset$, we may assume that $G\left(I^{i+1}\right) \cap F=\emptyset$. Let $\eta=$ $\min [\delta, \rho(K, X-N(K))]$. According to $(2 . n)$, there exists a map $f^{\prime}: I^{i+1} \rightarrow N_{\eta}(J)$ such that $\rho\left(G, f^{\prime}\right)<\varepsilon \circ G$. Check that $f^{\prime}\left(I^{i+1}\right) \subset N_{\eta}(J) \cap N_{2 \delta}$ and therefore

$$
f^{\prime}\left(I^{i+1}\right) \subset N_{\eta}(K) \subset N(K)
$$

Also, $f^{\prime} \mid S^{i}$ is $\gamma$-close to $G \mid S^{i}$; thus both $f^{\prime} \mid S^{i}$ and $G \mid S^{i}$ are homotopic in $N(K)$ and this shows that $f$ is null-homotopic in $N(K)$.

$F$ is proximately $L C^{n-1}$ in $X$ implies (2.n). The proof uses techniques found in $\left[\mathbf{C u}_{\mathbf{1}}\right]$. Since the proof is rather cumbersome and since (iii) suffices for our purposes in $\S 4$, we delete the proof. We note, however, that the proof of this result is a straightforward, though somewhat technical modification of the proof of (iii).

(iii) First, the fact that the reverse implication is false follows from an example of van Mill $[\mathbf{v M}]$ of a boundary set in the Hilbert cube containing no arcs. The proof for $n=0$ follows from (i) as every nowhere locally compact space satisfies the discrete 0 -cells property. The remainder of this section is devoted to a proof of (iii) in case $n$ is finite and nonzero.

If $n \in \mathbf{N}$ and $F$ is $L C^{n-1}$ in $X$, then condition (2.n) holds. Let $\bar{\varepsilon}: X-F \rightarrow$ $(0, \infty)$ be a map. We first replace the map $\bar{\varepsilon}$ by a map $\varepsilon$ that is defined on all of $X$. 
LEMMA. There is a map $\varepsilon: X \rightarrow[0,1]$ such that $0<\varepsilon(x)<\bar{\varepsilon}(x)$ for each $x \in X-F$.

PROOF. For each $x \in X-F$, choose an open neighborhood $U_{x}$ of $x$ in $X$ such that $\bar{\varepsilon}\left(U_{x}-F\right)$ is bounded away from 0 and let $\varepsilon_{x}=\inf \bar{\varepsilon}\left(U_{x}-F\right)>0$. Let $Y=\bigcup\left\{U_{x} \mid x \in X-F\right\}$, an open subset of $X$, and note that $X-Y \subset F$. For each $x \in X-F$, define $f_{x}: Y \rightarrow[0,1]$ via $f_{x}(y)=0$ if $y \notin U_{x}$ and $f_{x}(y)=\min \left\{\varepsilon_{x}, 1\right\}$ if $y \in U_{x}$. Each $f_{x}$ is a lower semicontinuous function on $Y$ and thus the function $f(y)=\sup \left\{f_{x}(y) \mid x \in X-F\right\}$ is lower semicontinuous on $Y$ [Wi, 7K.1]. Note that $0<f(y) \leq 1$ for each $y \in Y$ and $f(x) \leq \bar{\varepsilon}(x)$ for each $x \in X-F$. Theorem $4.3[\mathrm{Du}$, p. 171] applies to give a continuous function $\phi: Y \rightarrow[0,1]$ so that $0<\phi(y)<f(y)$ for each $y \in Y$.

Assuming $X \neq Y$, since $X-Y$ is a closed $G_{\delta}$ subset of $X$, there is a Urysohn function $\psi: X \rightarrow[0,1]$ with $\psi^{-1}(0)=X-Y$. Define $\varepsilon: X \rightarrow[0,1]$ by

$$
\varepsilon(x)= \begin{cases}\psi(x) \cdot \phi(x), & x \in Y ; \\ 0, & x \in X-Y .\end{cases}
$$

Clearly, $0<\varepsilon(x)<\bar{\varepsilon}(x)$ for each $x \in X-F$, and it is easy to check that $\varepsilon$ is continuous on $X$.

Throughout the remainder of this proof, $\varepsilon$ denotes the map of the lemma and $Y$ denotes the open subset of $X$ containing $X-F$ that appears in the proof of the lemma. Note that $\varepsilon(y)>0$ for each $y \in Y, \varepsilon^{-1}(0)=X-Y$, and, since $Y$ is an open subset of the locally compact separable metric space $X, Y$ is a locally compact separable metric space and as such is also $\sigma$-compact.

Write $Y=\bigcup_{i} \sigma_{i}$ where each $\sigma_{i}=\mathrm{Cl}_{Y}\left(\tau_{i}\right)$ is compact where $\tau_{i}$ is an open subset of $Y$ and, for each $i \in \mathbf{N}, \sigma_{i} \subset \tau_{i+1}$. Let $\sigma_{0}=\emptyset$. For each $i \in \mathbf{N}$, let $\varepsilon_{i}=2^{-i} \min \left[\varepsilon\left(\sigma_{i}\right)\right]$ and note that $\varepsilon_{1}>\varepsilon_{2}>\cdots>0$ and $\varepsilon_{i} \rightarrow 0$ as $i \rightarrow \infty$. Let $\delta_{i}(n+1)=\varepsilon_{i}$ and use the fact that each $\sigma_{i}$ is compact and $F$ is $L C^{n-1}$ in $X$ to choose for each $i \in \mathbf{N}, \delta_{i}(n)>0$ so small that if $\alpha: \partial I^{n} \rightarrow \tau_{i} \cap F$ and $\operatorname{diam} \alpha\left(\partial I^{n}\right)<\delta_{i}(n)$, then there exists an extension $\beta: I^{n} \rightarrow \tau_{i+1} \cap F$ of $\alpha$ so that

$$
\operatorname{diam} \beta\left(I^{n}\right)<\frac{1}{3} \delta_{i+2}(n+1) .
$$

Force the $\delta_{i}(n)$ 's to have the further property that $\delta_{i}(n)>\delta_{i+1}(n)$ and notice that $\delta_{i}(n) \rightarrow 0$ as $i \rightarrow \infty$. Continue in this manner and obtain positive constants $\delta_{i}(j)$ for $i \in \mathbf{N}$, and $j=1, \ldots, n+1$ that satisfy:

(1) $\delta_{i}(j) \rightarrow 0$ monotonically as $i \rightarrow \infty$ for each $j=1, \ldots, n+1$,

(2) $\delta_{i}(j-1)<\frac{1}{3} \delta_{i+2}(j)$ for $i=1,2, \ldots$ and $j=2, \ldots, n+1$,

(3) $\delta_{i}(n+1)=\varepsilon_{i} \leq \frac{1}{2} \min \left[\varepsilon\left(\sigma_{i}\right)\right]$ for $i \in \mathbf{N}$,

(4) if $\alpha: \partial I^{j} \rightarrow \tau_{i} \cap F$ and $\operatorname{diam} \alpha\left(\partial I^{j}\right)<\delta_{i}(j)$, then there exists an extension $\beta: I^{j} \rightarrow \tau_{i+1} \cap F$ of $\alpha$ so that $\operatorname{diam} \beta\left(I^{j}\right)<\frac{1}{3} \delta_{i+2}(j+1)$, for $j=1, \ldots, n$.

For each $i \in \mathbf{N}$, choose a finite collection of points $S_{i} \subset\left(\tau_{i}-\sigma_{i-1}\right) \cap F$ such that every point $x$ of $\sigma_{i}-\tau_{i-1}$ lies within $\frac{1}{3} \delta_{i+1}(1)$ of some element $s$ of $S_{i}-\{x\}$ and let $S_{0}=\emptyset$. 
Let $K$ be the collection of simplices whose vertices are the points of $\bigcup_{i} S_{i}$ and such that $\left\langle s_{0}, \ldots, s_{p}\right\rangle \in K$ for vertices $s_{0}, \ldots, s_{p}$ provided the following properties are satisfied:

(i) $p \leq n$,

(ii) $\left\{s_{0}, \ldots, s_{p}\right\} \subset S_{m-1} \cup S_{m}$ with some $s_{i} \in S_{m}$ for some $m \in \mathbf{N}$,

(iii) $\rho\left(s_{i}, s_{j}\right)<\delta_{m}(1)$ for $0 \leq i, j \leq p$ and $m$ as in (ii).

The reader may verify that (i) through (iii) define $K$ as a locally finite $n$-dimensional abstract simplicial complex.

There is an obvious map $\nu_{0}: K^{(0)} \rightarrow J_{0} \subset F$ onto the closed subset $J_{0}=\bigcup_{i} S_{i}$ of $Y$. Given $\left\langle s_{0}, s_{1}\right\rangle \in K^{(1)}$ suppose that $s_{0}, s_{1} \in S_{m-1} \cup S_{m}$ with one of $s_{0}$ or $s_{1}$ in $S_{m}$. Then $\rho\left(s_{0}, s_{1}\right)<\delta_{m}(1)$ and $s_{0}, s_{1} \in \tau_{m} \cap F$ so that $(*)(4)$ applies to produce a $\operatorname{map} \beta:\left\langle s_{0}, s_{1}\right\rangle \rightarrow \tau_{m+1} \cap F$ so that $\operatorname{diam} \beta\left(\left\langle s_{0}, s_{1}\right\rangle\right)<\frac{1}{3} \delta_{m+2}(2)$ and $\beta\left(s_{i}\right)=s_{i}$ for $i=1,2$. In this way extend $\nu_{0}$ to obtain $\nu_{1}: K^{(1)} \rightarrow F$. Note that if $\left\langle s_{0}, s_{1}, s_{2}\right\rangle \in$ $K^{(2)}$ then $\operatorname{diam} \nu_{1}\left(\left\langle s_{i}, s_{j}\right\rangle\right)<\frac{1}{3} \delta_{m+1}(2)$ for $i, j \in\{0,1,2\}$ where $s_{0}, s_{1}, s_{2} \in S_{m-1} \cup$ $S_{m}$ and at least one of $s_{0}, s_{1}, s_{2}$ is in $S_{m}$. Thus diam $\nu_{1}\left(\partial\left\langle s_{0}, s_{1}, s_{2}\right\rangle\right)<\delta_{m+1}(2)$ and $\nu_{1}\left(\partial\left\langle s_{0}, s_{1}, s_{2}\right\rangle\right) \subset \tau_{m+1} \cap F$. $(*)(4)$ applies again to give an extension $\nu_{2}$ of $\nu_{1} \mid \partial\left\langle s_{0}, s_{1}, s_{2}\right\rangle$ to $\left\langle s_{0}, s_{1}, s_{2}\right\rangle$ so that $\operatorname{diam} \nu_{2}\left(\left\langle s_{0}, s_{1}, s_{2}\right\rangle\right)<\frac{1}{3} \delta_{m+3}(3)$. We continue through $(n-1)$-steps and obtain a map $\nu_{n}: K^{(n)} \rightarrow F$ so that $\nu_{n}\left(\left\langle s_{0}, \ldots, s_{n}\right\rangle\right)$ has diameter less than $\frac{1}{3} \delta_{m+n+1}(n+1) \leq \varepsilon_{m+n+1}$ if $\left\{s_{0}, \ldots, s_{n}\right\} \subset S_{m-1} \cup S_{m}$ and at least one of $s_{0}, \ldots, s_{n}$ is in $S_{m}$. Let $\nu=\nu_{n}$.

We claim that $\nu(K)$ is a closed subset of $Y$. Indeed, for each $m \in \mathbf{N}$, denote by $K_{m}$ the finite subcomplex of $K$ which consists of all simplices of $K$ all of whose vertices lie in $S_{1} \cup S_{2} \cup \cdots \cup S_{m-1}$. Given a positive integer $j$, choose $m>j+1$ so large that $\varepsilon_{m+n+1}<\frac{1}{2} \rho\left(\sigma_{j}, Y-\tau_{j+1}\right)$ and suppose that $\left\langle s_{0}, \ldots, s_{p}\right\rangle \in$ $K-K_{m}$ and $\left\{s_{0}, \ldots, s_{p}\right\} \subset S_{k-1} \cup S_{k}$ with say $s_{0} \in S_{k}$ where $k \geq m$. Then $\operatorname{diam} \nu\left(\left\langle s_{0}, \ldots, s_{p}\right\rangle\right)<\varepsilon_{k+n+1} \leq \varepsilon_{m+n+1}<\frac{1}{2} \rho\left(\sigma_{j}, Y-\tau_{j+1}\right) \leq \frac{1}{2} \rho\left(\sigma_{j}, Y-\tau_{k-1}\right)$ and since $\nu\left(s_{0}\right)=s_{0} \in Y-\tau_{k-1}, \nu\left(\left\langle s_{0}, \ldots, s_{p}\right\rangle\right) \cap \sigma_{j}=\emptyset$. We have shown that $\nu(K) \cap \sigma_{j}=\nu\left(K_{m}\right) \cap \sigma_{j}$, which is compact since $K_{m}$ is compact and this implies that $\nu(K)$ is closed in $Y$.

Let $J=\mathrm{Cl}_{X}(\nu(K))$. Since $\nu(K) \subset F \cap Y$ is closed in $Y$ and $X-Y \subset F, J \subset F$. In fact, it follows easily that $J=(X-Y) \cup \nu(K)$. The next lemma shows that $J$ " $\varepsilon$-absorbs" $n$-cells from $X-F$ and this completes the proof of Theorem 3.6.

LEMMA. Let $f: I^{n} \rightarrow X-F$ be a map. Then there exists a map $g: I^{n} \rightarrow J$ such that $\rho(f, g)<\varepsilon \circ f$.

PROOF. Choose $m$ so large that $f\left(I^{n}\right) \subseteq \tau_{m}$ and choose a triangulation $T$ of $I^{n}$ such that each $\lambda \in T$ satisfies diam $f(\lambda)<\frac{1}{3} \delta_{m+1}(1)$ and $f(\lambda) \subset \tau_{k}-\sigma_{k-2}$ for some integer $k$ with $m \geq k \geq 2$. For each vertex $t \in T^{(0)}$, there is a unique integer $k$ with $m \geq k \geq 1$ such that $f(t) \in \tau_{k}-\tau_{k-1}$. For each such vertex $t$, choose $s(t) \in S_{k}$ which lies within $\frac{1}{3} \delta_{k+1}(1)$ of $f(t)$ (see the definition of $S_{k}$ ) and define $\theta: T^{(0)} \rightarrow K^{(0)}$ via $\theta(t)=s(t)$. Suppose that $\lambda=\left\langle t_{0}, \ldots, t_{p}\right\rangle \in T$ where, of course, $p \leq n$. There is an integer $k$ with $m \geq k \geq 2$ such that $\left\{f\left(t_{0}\right), \ldots, f\left(t_{p}\right)\right\} \subset \tau_{k}-\sigma_{k-2}$ and thus, $\left\{\theta\left(t_{0}\right), \ldots, \theta\left(t_{p}\right)\right\} \subset S_{k-1} \cup S_{k}$ and we may assume without loss of generality that $\theta\left(t_{0}\right) \in S_{k}$. For $i$ and $j$ in $\{0, \ldots, p\}$ we have

$$
\begin{aligned}
\rho\left(\theta\left(t_{i}\right), \theta\left(t_{j}\right)\right) & \leq \rho\left(\theta\left(t_{i}\right), f\left(t_{i}\right)\right)+\rho\left(f\left(t_{i}\right), f\left(t_{j}\right)\right)+\rho\left(f\left(t_{j}\right), \theta\left(t_{j}\right)\right) \\
& \leq \frac{1}{3} \delta_{k}(1)+\frac{1}{3} \delta_{m+1}(1)+\frac{1}{3} \delta_{k}(1) \leq \delta_{k}(1) .
\end{aligned}
$$


Thus $\theta\left(t_{0}\right), \ldots, \theta\left(t_{p}\right)$ satisfy (i)-(iii) of the definition of $K$ and therefore $\left\langle\theta\left(t_{0}\right), \ldots, \theta\left(t_{p}\right)\right\rangle \in K$. This shows that $\theta$ sends the vertices of any simplex of $T$ to the vertices of some simplex of $K$ and therefore, we may extend $\theta$ linearly to obtain a simplicial map (still called $\theta$ ) $\theta: T \rightarrow K$.

Let $g: I^{n} \rightarrow J$ be given by $g=\nu \circ \theta$ and let $x \in \lambda \in T$ where $\lambda$ is an $n$-simplex. There is an integer $k$ with $m \geq k \geq 2$ such that $f(\lambda) \subset \tau_{k}-\sigma_{k-2}$ and therefore $\operatorname{diam} g(\lambda)=\operatorname{diam} \nu(\theta(\lambda))<\varepsilon_{k+n}$ since $\theta\left(\lambda^{(0)}\right) \subset S_{k-1} \cup S_{k}$. Let $t \in \lambda^{(0)}$. Then

$$
\begin{aligned}
\rho(f(x), g(x)) & \leq \rho(f(x), f(t))+\rho(f(t), g(t))+\rho(g(t), g(x)) \\
& \leq \frac{1}{3} \delta_{m+1}(1)+\frac{1}{3} \delta_{k}(1)+\varepsilon_{k+n} .
\end{aligned}
$$

Properties $(*)(1)$ through $(*)(3)$ along with the fact that $k \leq m$ apply to show that $\delta_{m+1}(1)<\delta_{m+1}(n+1)=\varepsilon_{m+1}<\varepsilon_{k}, \delta_{k}(1)<\delta_{k}(n+1)=\varepsilon_{k}$, and $\varepsilon_{k+n}<\varepsilon_{k}$. Thus property $(*)(3)$ shows that

$$
\rho(f(x), g(x))<2 \varepsilon_{k} \leq \min \left[\varepsilon\left(\sigma_{k}\right)\right] \leq \varepsilon(f(x)) .
$$

4. Discrete cells properties and products of dendrites. Throughout this section $A$ denotes an arbitrary element of $A$ and $\bar{A}$ denotes the element of $\bar{A}$ from which $A$ arises. In the following sense, $\bar{A}^{n+1}$ can be thought of as a "finite dimensional Hilbert cube." Both $\bar{A}^{n+1}$ and the Hilbert cube $I^{\infty}$ are compact absolute retracts and both $A^{n+1}$ and the pseudo-interior $s$ of the Hilbert cube are nowhere locally compact topologically complete absolute retracts that arise as the complements of dense $\sigma$ - $Z$-sets in $\bar{A}^{n+1}$ and $I^{\infty}$, respectively. Compact subsets of $A^{n+1}$ are $Z_{n}$-sets in both $A^{n+1}$ and $\bar{A}^{n+1}$ (Corollary 2.10) and compact subsets of $s$ are $Z_{\infty}$-sets in both $s$ and $I^{\infty} \cdot \bar{A}^{n+1}$ satisfies the disjoint $n$-cells property (Corollary 2.3) and as such is a universal embedding space for $n$-dimensional compacta while $I^{\infty}$ satisfies the disjoint Hilbert cubes property and is a universal embedding space for compacta. $A^{n+1}$ satisfies the discrete $n$-cells property (Corollary 4.3 ) and as such is a universal (closed) embedding space for topologically complete separable $n$-dimensional spaces while $s$ satisfies the discrete approximation property and is a universal (closed) embedding space for topologically complete separable spaces. Also, $\bar{A}^{\infty} \approx I^{\infty}$ and $A^{\infty} \approx s$. For these reasons we call $A^{n+1}$ the pseudo-interior of $\bar{A}^{n+1}$ and $F_{n}=\bar{A}^{n+1}-A^{n+1}$ the pseudo-boundary of $\bar{A}^{n+1}$.

Recall that $F_{0}=\bar{A}-A$ is a dense $\sigma$-compact collection of endpoints of $\bar{A}$. The main result of this section is Theorem 4.1 and this allows us to conclude that $A^{n+1}$ satisfies the discrete $n$-cells property.

THEOREM 4.1. Let $F$ be a dense subset of a space $X$ and let $n \in \mathbf{N} \cup\{0\}$. If $F$ is $L C^{n-1}$ in $X$ and $X$ is $L C^{n}$, then $(F \times \bar{A}) \cup\left(X \times F_{0}\right)$ is $L C^{n}$ in $X \times \bar{A}$.

COROLLARY 4.2. Under the hypotheses of Theorem 4.1, if in addition $F$ is a $\sigma$ - $Z$-set in $X$ and $X$ is a locally compact separable $L C^{n+k-1}$-space for some $k \in \mathbf{N}$, then $(X-F) \times A^{k}$ satisfies the discrete $(n+k)$-cells property.

Proof. Since $X$ is $L C^{n+k-1}, X \times \bar{A}^{k}$ is $L C^{n+k-1}$. Induction and Theorem 4.1 reveal that the dense $\sigma$ - $Z$-set $\left(F \times \bar{A}^{k}\right) \cup\left(X \times\left(\bar{A}^{k}-A^{k}\right)\right)$ is $L C^{n+k-1}$ in $X \times \bar{A}^{k}$ and Theorem 3.6(i) and (iii) then apply to show that $(X-F) \times A^{k}$ satisfies the discrete $(n+k)$-cells property.

Corollary 4.3 is stated in the introduction. 
PROOF OF COROLlaRY 4.3. The proof for $n=0$ is left to the reader as every nowhere locally compact space satisfies the discrete 0-cells property. If $W$ and $B$ are connected and therefore path connected open subsets of $\bar{A}$, then since $F_{0}$ is dense in $\bar{A},(W \times B) \cap F_{1}$ is path connected where $F_{1}=\left(\bar{A} \times F_{0}\right) \cup\left(F_{0} \times \bar{A}\right) \subset \bar{A}^{2}$. This implies that $F_{1}$ is $L C^{0}$ in $\bar{A}^{2}$ and according to Theorem $3.6, A^{2}$ satisfies the discrete 1-cells property. The fact that $A^{n+1}$ satisfies the discrete $n$-cells property for $n$ finite is now a direct consequence of Corollary 4.2. For $n=\infty$, Corollary 4.2 applies to show that $A^{\infty}$ satisfies the discrete $n$-cells property for all $n \in \mathbf{N}$ and, in this setting where $A^{\infty}$ is the complement of a dense $\sigma$ - $Z$-set in the compact ANR $\bar{A}^{\infty}$, this is equivalent to the discrete approximation property (= discrete $\infty$-cells property) $\left[\mathbf{B o}_{2}\right]$.

Actually, Theorem 4.1 applies to show that the pseudo-boundary $F_{n}=\bar{A}^{n+1}$ $A^{n+1}$ is $L C^{n-1}$ in $\bar{A}^{n+1}$ since $F_{n}=\left(F_{n-1} \times \bar{A}\right) \cup\left(\bar{A}^{n} \times F_{0}\right)$ and this guarantees that $A^{n+1}$ satisfies the discrete $n$-cells property. The remainder of this section is devoted to a proof of Theorem 4.1.

PROOF OF THEOREM 4.1. If $W$ and $B$ are path connected open subsets of $X$ and $\bar{A}$, respectively, then since $F$ is dense in $X$ and $F_{0}$ is dense in $\bar{A},(W \times$ $B) \cap\left[(F \times \bar{A}) \cup\left(X \times F_{0}\right)\right]$ is path connected. Since $X$ is $L C^{0}$, this ensures that $(F \times \bar{A}) \cup\left(X \times F_{0}\right)$ is $L C^{0}$ in $X \times \bar{A}$.

Assume that $n>0$. Let $x \in X$ and let $W^{\prime}$ be a neighborhood of $x$ in $X$ and $B$ a contractible open subset of $\bar{A}$. Since $X$ is $L C^{n}$, choose a neighborhood $W$ of $x$ in $X$ such that maps $S^{i} \rightarrow W$ for $0 \leq i \leq n$ are null-homotopic in $W^{\prime}$. It is sufficient to show that every map $S^{i} \rightarrow(W \times B) \cap F_{*}$ for $0 \leq i \leq n$ is null-homotopic in $\left(W^{\prime} \times B\right) \cap F_{*}$ where $F_{*}=(F \times \bar{A}) \cup\left(X \times F_{0}\right)$. We show this for $i=n$, the remaining cases being similar.

Let $f: \partial I^{n+1} \rightarrow(W \times B) \cap F_{*}$ be a map and $p_{1}: X \times \bar{A} \rightarrow X$ and $p_{2}: X \times$ $\bar{A} \rightarrow \bar{A}$ be the projections. Let $g: I^{n+1} \rightarrow W^{\prime} \times B$ be any extension of $f$ to the $(n+1)$-cell $I^{n+1}$ and choose collections of product open subsets of $W^{\prime} \times B$, say $\mathcal{V}_{0}, \mathcal{U}_{0}, \ldots, \mathcal{V}_{n-1}, \mathcal{U}_{n-1}, \mathcal{V}, \mathcal{U}$, such that the following hold:

(i) for each $U \in \mathcal{U}, \rho\left(g\left(\partial I^{n+1}\right), U\right)>\operatorname{diam} U$ where $\rho$ denotes a metric on $X \times \bar{A}$,

(ii) $V_{0}$ is a cover of $g\left(I^{n+1}\right)-g\left(\partial I^{n+1}\right)$,

(iii) $\mathcal{V}_{0}<U_{0}<^{*} \mathcal{V}_{1}<\cdots<^{*} \mathcal{V}_{n-1}<U_{n-1}<^{*} \mathcal{V}<U$ where $<^{*}$ denotes starrefinement,

(iv) for each $j=0,1, \ldots, n-1$ and for each $V \in \mathcal{V}_{j}$, there exists a $U \in \mathcal{U}_{j}$ containing $V$ such that $\pi_{j}\left(p_{1}(V) \cap F\right) \rightarrow \pi_{j}\left(p_{1}(U) \cap F\right)$ is the zero homomorphism, and for each $V \in \mathcal{V}$, there exists a $U \in \mathcal{U}$ containing $V$ such that $\pi_{n}\left(p_{1}(V)\right) \rightarrow$ $\pi_{n}\left(p_{1}(U)\right)$ is the zero homomorphism.

This choice is possible since $X$ is $L C^{n}$ and $F$ is $L C^{n-1}$ in $X$. (Choose these covers in reverse order, starting with $\mathcal{U}$ and ending with $\mathcal{V}_{0}$.)

Let $T$ be a locally finite triangulation of $I^{n+1}-g^{-1} g\left(\partial I^{n+1}\right)$ such that $T$ refines $g^{-1}\left(\mathcal{V}_{0}\right)$ and such that diameters of simplices go to zero near $g^{-1} g\left(\partial I^{n+1}\right)$. For each simplex $\sigma \in T$, choose $V_{\sigma} \in \mathcal{V}_{0}$ such that $g(\sigma) \subset V_{\sigma}$ and for each vertex $t \in T^{(0)}$, choose a point $\theta(t)$ in $F$ which lies in $\bigcap\left\{p_{1}\left(V_{\sigma}\right) \mid t \in \sigma^{(0)}\right\}$ and within $\rho\left(g(t), g\left(\partial I^{n+1}\right)\right)$ of $p_{1}(g(t))$. Thus obtain a map $\theta: T^{(0)} \rightarrow W^{\prime} \cap F$ such that if $t_{i} \in T^{(0)}$ and $t_{i} \rightarrow x \in g^{-1} g\left(\partial I^{n+1}\right)$, then $\theta\left(t_{i}\right) \rightarrow p_{1}(g(x))$.

Since for each $\sigma \in T^{(1)}, \theta(\partial \sigma) \subset p_{1}(V) \cap F$ for some $V \in \mathcal{V}_{0}$, (iv) allows us to extend $\theta$ to $T^{(1)}$ so that by (iii), if $\sigma \in T^{(2)}$, then $\theta(\partial \sigma) \subset p_{1}(V) \cap F$ for some 
$V \in \mathcal{V}_{1}$. We continue to extend $\theta$ on higher dimensional skeleta of $T$ until we obtain a map $\theta: T^{(n)} \rightarrow W^{\prime} \cap F$ that is limited by $p_{1}(\mathcal{V})$, that is, for each $(n+1)$-simplex $\Delta$ in $T, \theta(\partial \Delta)$ is contained in $p_{1}(V)$ for some $V \in \mathcal{V}$. Let $\psi: T^{(n)} \rightarrow W^{\prime} \times B$ be the map $\psi=\left(\theta, p_{2} \circ g \mid T^{(n)}\right)$ and extend $\psi$ to $T$ by extending on each $(n+1)$-simplex $\Delta$ of $T$ using (iv) via the trick used in the proof of Theorem 2.1 so that $\psi(\Delta) \subset U$ for some $U \in \mathcal{U}$ and

$$
p_{1} \psi(c(\partial \Delta))=p_{1}(\psi(\partial \Delta)) \subset F
$$

and $p_{2} \psi(\Delta-c(\partial \Delta)) \in F_{0}$ where $c(\partial \Delta)$ denotes a collar on $\partial \Delta$. Since if $t_{i} \in T^{(0)}$ and $t_{i} \rightarrow x \in g^{-1} g\left(\partial I^{n+1}\right), \psi\left(t_{i}\right)=\left(\theta\left(t_{i}\right), p_{2} g\left(t_{i}\right)\right) \rightarrow g(x)$, and since $\psi$ is limited by $U$, (i) allows us to extend $\psi$ to $g^{-1} g\left(\partial I^{n+1}\right)$ via $g$ to obtain a map $h: I^{n+1} \rightarrow W^{\prime} \times B$ given by $h=g$ on $g^{-1} g\left(\partial I^{n+1}\right)$ and $h=\psi$ otherwise. Notice that $h=f$ on $\partial I^{n+1}$.

Given $x \in I^{n+1}$, there are three possibilities: either $h(x)=g(x) \in F_{*}$ (for $\left.x \in g^{-1} g\left(\partial I^{n+1}\right)\right), p_{1} h(x)=p_{1} \psi(x) \in F($ for $x \in c(\partial \Delta))$, or $p_{2} h(x)=p_{2} \psi(x) \in F_{0}$ (for $x \in \Delta-c(\partial \Delta)$ ). Thus $h\left(I^{n+1}\right) \subset F_{*}$ and this implies since $h \mid \partial I^{n+1}=f$ that $f$ is null-homotopic in $\left(W^{\prime} \times B\right) \cap F_{*}$. This completes the proof.

\section{REFERENCES}

[ACM] R. D. Anderson, D. W. Curtis and J. van Mill, A fake topological Hilbert space, Trans. Amer. Math. Soc. 272 (1982), 311-321.

[Bo 1 P. L. Bowers, Applications of general position properties of dendrites to Hilbert space topology, Ph.D. Dissertation, Univ. of Tennessee, 1983.

$\left[\mathbf{B o}_{2}\right] \ldots$, Discrete cells properties in the boundary set setting, Proc. Amer. Math. Soc. (to appear).

[Ca] J. W. Cannon, Shrinking cell-like decompositions of manifolds. Codimension three, Ann. of Math. (2) 110 (1979), 83-112.

[Ch] T. A. Chapman, Lectures on Hilbert cube manifolds, CBMS Regional Conf. Ser. in Math., no. 28, Amer. Math. Soc., Providence, R.I., 1976.

$\left[\mathrm{Cu}_{1}\right]$ D. W. Curtis, Boundary sets in the Hilbert cube, preprint.

$\left[\mathbf{C u}_{2}\right]$ _ Preliminary report, boundary sets in the Hilbert cube and applications to hyperspaces, preprint.

[Da] R. J. Daverman, Detecting the disjoint disks property, Pacific J. Math. 93 (1981), 277-298.

[DW] R. J. Daverman and J. J. Walsh, Cech homology characterizations of infinite dimensional manifolds, Amer. J. Math. 103 (1981), 411-435.

[DT] T. Dobrowolski and H. Torunczyk, On metric linear spaces homeomorphic to $l_{2}$ and compact convex subsets homeomorphic to $Q$, Bull. Acad. Polon. Sci. 27 (1979), 883-886.

[Du] J. Dugundji, Topology, Allyn and Bacon, Boston, Mass., 1966.

[Ed] R. D. Edwards, Approximating certain cell-like maps by homeomorphisms, Abstract preprint. See also Notices Amer. Math. Soc. 24 (1977), A649, \#751-G5.

[HW] W. Hurewicz and H. Wallman, Dimension theory, Princeton Univ. Press, Princeton, N.J., 1969.

[vM] J. van Mill, $A$ boundary set for the Hilbert cube containing no arcs, Fund. Math. (to appear).

[Qu] F. Quinn, Ends of maps and applications, Ann. of Math. (2) 110 (1979), 275-331.

[Si] K. Sieklucki, A generalization of a theorem of K. Borsuk concerning the dimension of ANR-sets, Bull. Acad. Polon. Sci. 10 (1962), 433-436.

[To 1 ] H. Torunczyk, On CE-images of the Hilbert cube and characterization of $Q$-manifolds, Fund. Math. 106 (1980), 31-40.

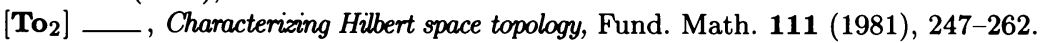

[Wi] S. Willard, General topology, Addison-Wesley, Reading, Mass., 1970.

Department of Mathematics, Florida State University, Tallahassee, FlorIDA 32306 